\title{
Polarized Cluster Dynamics at the Paraelectric to Ferroelectric Phase Transition in $\mathrm{BaTiO}_{3}$
}

\author{
Marek Paściak, ${ }^{\dagger, \downarrow}$ Salah Eddine Boulfelfel, ${ }^{\S, \|}$ and Stefano Leoni ${ }^{*, \S, \perp}$ \\ Research School of Chemistry, Australian National University, Canberra ACT 0200, Australia, Institute of Low \\ Temperature and Structure Research, Polish Academy of Sciences, P.O. Box 1410, 50-950 Wroctaw, Poland, \\ Max-Planck-Institut für Chemische Physik fester Stoffe, Nöthnitzer Strasse 40, 01187 Dresden, Germany, \\ Department of Geosciences, Stony Brook University, Stony Brook, New York 11794-2100, United States, and \\ Physikalische Chemie, Technische Universität Dresden, 01062 Dresden, Germany
}

Received: July 5, 2010; Revised Manuscript Received: October 20, 2010

\begin{abstract}
The mechanism of the prototype ferroelectric phase transition in $\mathrm{BaTiO}_{3}$ is a matter of intense debate and to a large extent still wrapped in mystery. Precursor phenomena in the form of polarized clusters in paraelectric $\mathrm{BaTiO}_{3}$ are by now believed to represent a key step into the ferroelectric phenomenon. The determination of a slower dynamics of cluster polarization flipping along with a faster order-disorder Ti hopping mode among $\langle 111\rangle$ off-center sites suggests coexistence, instead of mutual exclusion, of displacive and order-disorder types, initially proposed as distinct models. However, no clear picture of the transition state has been proposed so far, which is able to provide insight into the coexistence of the paraelectric and ferroelectric phenomena. Here, by means of a dedicated molecular dynamics approach, we provide a detailed atomistic picture of intermediate regions along the transition. Therein, different time and length scales coexist as they characterize different portions of the same material. From an imbalance of dynamically and more statically polarized clusters in this highly inhomogeneous intermediate, a symmetry breaking step naturally results. Further, we find that ferroelectric nanodomains may host antiferroelectric defects, which appear as an intrinsic feature of the growing $\mathrm{BaTiO}_{3}$ ferroelectric material.
\end{abstract}

\section{Introduction}

Ferroelectric materials are broadly employed as capacitors and memory materials. However, even for the classical compound $\mathrm{BaTiO}_{3}$ (BTO), microscopic details on the cubic paraelectric (PE) and on the ferroelectric (FE) phases and their transformations remain puzzling. In $\mathrm{BaTiO}_{3}$, the off-center displacement of $\mathrm{Ti}$ atoms creates a net electric dipole moment. In terms of domain growth, a critical size ${ }^{1}$ may thus be required for the ferroelectric state to be stable with respect to the competing electrostatic energy, caused by an asymmetric charge distribution. Recently, theoretical ${ }^{2,3}$ and experimental ${ }^{4}$ evidence $^{2}$ on fundamental size limits in ferroelectricity has been provided.

Two classical models for ferroelectricity exist, the displacive ${ }^{5}$ and the order-disorder model, ${ }^{6-8}$ which individually account for some experimental facts. ${ }^{9}$ In the displacive model, the softening of TO phonon modes detected by neutron scattering ${ }^{10}$ are microscopically connected to Ti leaving the center of the oxygen octahedra at the transition onset. In the meanwhile, the two models are considered limiting cases (pertinent to different time scales) of the same ferroelectric phenomenon. ${ }^{11-13}$ Recent $\mathrm{THz}$ spectroscopy and IR reflectivity experiments show that the soft mode coexists in $\mathrm{BaTiO}_{3}$ with a central mode, the latter being evidence of relaxational dynamics and thus of order-disorder behavior. ${ }^{14,15}$ Further, direct imaging experiments are disclosing the complex potential landscape of nucleation in ferroelectrics ${ }^{16}$ and pointing out the reduced role of thermal fluctuations on

* To whom correspondence should be addressed. E-mail: stefano.leoni@ chemie.tu-dresden.de.

† Australian National University.

* Polish Academy of Sciences.

$\S$ Max-Planck-Institut für Chemische Physik fester Stoffe.

"Stony Brook University.

$\perp$ Technische Universität Dresden. ferroelectric response. ${ }^{17}$ Simulations ${ }^{18-20}$ and experiments ${ }^{21-24}$ indicate the existence of dynamic polar clusters already in the paraelectric, cubic phase. As precursors to the phase transition, the appearance of polarized clusters characterize this region. Their critical decay around the Curie temperature has been the object of accurate measurements, recently. ${ }^{25}$ Therein the mutual influence of polarization clusters is quantitatively characterized, as an additional indication of the complexity of the transforming paraelectric phase.

Molecular dynamics simulations have been successfully used in connection with ferroelectric transitions, ${ }^{26,27}$ as they allow the capture and description of phase transitions. In this work, to shed light on the intermediate region, we perform molecular dynamics (MD) simulations on the phase transition from PE cubic $(c)$ to FE tetragonal $(t)$ in BTO at equilibrium conditions $\left(T=T_{\mathrm{c}}\right)$. We are interested in capturing the complexity of the paraelectric/ferroelectric region by atomistic simulations, accounting for intermediate configurations in regions of coexistence of the two phenomena. We used transition path sampling molecular dynamics (TPSMD ${ }^{28,29}$ ) as a means to elucidate the mechanism of phase transitions in detail. ${ }^{29-32}$ Performing TPS MD entails collecting a large number of critical trajectories, the transition path ensemble, to achieve a complete, albeit sophisticated, characterization of the transition state. As such, it is tailor-made for approaching the problem of clustering dynamics in the paraelectric phase and subsequent ferrodomain growth in BTO across the ferroelectric phase transition. In the second part, the results of MD simulations on the cubictetragonal phase transition are coarse-grained into a Heisenberg spin-like model, which is used to compute domain formation for all ferroelectric transitions (cubic-tetragonal-orthorhombicrhombohedral) and to evaluate diffuse scattering (DS). 


\section{Methods}

2.1. Molecular Dynamics Simulations. Constant temperature, constant pressure classical molecular dynamics simulations were performed in the anisotropic NoT ensemble ${ }^{33}\left(T=T_{c}\right)$ using the polarizable shell interatomic potential of Sepliarsky et al. ${ }^{34}$ The shell potential model has proven to give a reliable description of the phonon spectrum and also of local displacements in BTO. As such, it does not bias the mechanism in any direction (order-disorder or displacive). TPSMD is an iterative process and requires an initial trajectory. ${ }^{29}$ The latter was chosen in a regime of Ti positions shifted off-center from the ideal cubic arrangement, corresponding to the displacive model. Our TPSMD simulations were organized along the following steps:

1. A first trajectory connecting FE (tetragonal) to PE (cubic) was obtained by time propagating an initial configuration. The latter was prepared with all Ti coherently off-center with respect to the oxygen octahedra.

2. A time slice was randomly selected from the previous trajectory. Therein, a perturbation in form of random momenta modifications was incorporated. This was done under strict conservation of linear and angular momenta.

3. A new trajectory was grown, propagating in both directions of time, in order to reach $\mathrm{PE}$ and FE states on both ends, respectively.

4. Momenta modifications were introduced on the new trajectory, and the procedure was iterated.

5. In case the trajectory failed to connect PE to FE, the new trajectory was rejected and a new perturbation move (step 4) was undertaken.

The initial regime (first trajectory) is quickly abandoned in the course of simulations, and a clear tendency to grow $\mathrm{Ti}-\mathrm{O}$ chains along the $c \rightarrow t$ transition appeared. In the course of TPSMD, a too narrow box would translate into an increased difficulty to stabilize the tetragonal configuration on chain growth. It is known that the choice of suitable box dimensions in finite size simulations (with periodic boundary conditions) is a critical move. Therefore, we used the intrinsic length rescaling indicated by the simulation itself to define an appropriate size of the simulation system. Successive enlargement of the box dimensions showed that a $12^{3}$ unit cells system is an adequate size to study polarized cluster dynamics and their evolution into the ferroelectric state. Shooting off new trajectories with this box size choice allows the observation of cluster formation and growth and does not affect the overall transition profile, such that both FE and PE states are well described. A representative trajectory resulted from integrating the equations of motion over $50 \mathrm{ps}$, with a time step of $0.2 \mathrm{fs}$. Completion of the transition was estimated based on order parameters. The simulations with an external static electric field were performed in a smaller box. As the field favors $t$ BTO, a $9^{3}$ unit cells simulation box suffices in this case.

2.2. Monte Carlo Simulations. The system of $50 \times 50 \times$ $50 \sigma$-vectors with periodic boundary conditions was used in Monte Carlo simulations based on a spin-like Heisenberg Hamiltonian (see section 3.4 for details). We employed mixed single-spin (Metropolis algorithm ${ }^{35}$ ) and cluster (Wolff algorithm $^{36}$ ) dynamics for better equilibration. The first phase of calculation comprised fitting of model parameter runs. The transverse and longitudinal correlation lengths (for up to third nearest neighbor) extracted from MD-derived structures (paraelectric phase) were used as a fitting basis. Subsequent simulations within a given phase involved up to $10^{5} \mathrm{MC}$ steps (each step consisted of one spin and one cluster flip).
2.3. Diffuse Scattering. Diffraction patterns were calculated for structures constructed on the basis of well equilibrated systems resulting from MC simulation. Each vector $\sigma$ was backtransformed to relative $\mathrm{Ti}-\mathrm{O}$ shift. Diffuse scattering intensities were calculated and displayed using DIFFUSE package. ${ }^{37}$ The transverse correlation of the $x(y)$ component of displacement is calculated as follows: $(1 / N) \sum_{i, j} \sigma_{i}^{\xi} \cdot \sigma_{j}^{\xi}, \xi=x, y . \vec{\sigma}$ is the vector of Ti displacement. The indices $i, j$ refer to neighbor cells in the transverse directions.

\section{Results and Discussion}

The distinction between cubic and tetragonal structures was ensured by several order parameters, evaluated upon monitoring cell parameter changes and polarization, and upon collecting time-averaged statistics over $\mathrm{Ti}-\mathrm{O}$ relative displacements. Depending on the time-resolution of the experimental method, different characteristics of the phase transition appear. Upon inspecting the calculated trajectories, both PE and FE BTO phases host $\mathrm{Ti}$ displacements, connected over oxygen into dynamic $(\mathrm{Ti}-\mathrm{O})_{m}$ chains. ${ }^{18}$ The Ti environment is essentially rhombohedral in the FE phase (as in all phases of BTO, see section 3.4), due to the contraction of three $\mathrm{Ti}-\mathrm{O}$ bonds and elongation of the other three. In PE BTO Ti shifts are along $\langle 111\rangle$, while in FE BTO they deviate from the closest $\langle 111\rangle$ directions by $12.5^{\circ}$, in agreement with XAFS results, ${ }^{42} 11.8 \pm$ $1.1^{\circ}$, and comparable with the $10.8^{\circ}$ based on the AFE/FE model. ${ }^{38}$ Instantaneous projections of Ti displacements indicate $\langle 111\rangle$ shifts in both PE and FE phases, whereby upon time averaging they only survive in the $t$ phase, along [001].

3.1. Chains and Ferrodomain Growth. To monitor the progress of the ferroelectric transition, the time-averaged vector of Ti $\langle 111\rangle$ shifts needs be considered. A time window of 200 ps was considered. Therein, transverse correlation in the $x, y$ plane, and time autocorrelation of $\mathrm{Ti}-\mathrm{O}$ chain formations, were evaluated. Transverse correlation is an appropriate quantity to monitor the progress of domain growth as well as FE and AFE couplings, which appear as vector field features (cluster and domain shapes) in Figure 1a. The averaged $z$ displacements $(\delta z)$ are rendered in color in Figure 1 with respect to the polarization vector, red for upward $(+)$ and blue for downward $(-)$. The existence of polar clusters already in the PE phase $(0-8 \mathrm{ps})$ is clearly seen. The fast time scale of cluster aggregation/ decomposition is less than $1 \mathrm{ps}$, for a cluster size up to 2-3 unit cells of locally rhombohedral pattern, supporting recent experiments. ${ }^{23,24}$ However, setting in at $t \geq 8$ ps the dynamically correlated clusters grow larger and literally freeze into domains (Figure 1a), marked by a steep change of the time autocorrelation (Figure 2). Subsequently, the overall slowdown of the process continues $(15-30 \mathrm{ps})$ until a smooth landscape of $180^{\circ}$ polar domains is reached ( $t \geq 40 \mathrm{ps})$. A quantitative description of the transition in terms of order parameter changes is given in Figure 2. Relative variation of lattice parameters amounts to $c / a=1.01$, in agreement with the experimental value of $1.009 .{ }^{39}$ The value of spontaneous polarization due to the formation of domains is vanishing on the average, with a hidden tendency of the polarization $P_{z}$ component to drift. The nonzero value of the transverse correlation (Figure 2) along all three Cartesian axes already in the PE phase is indicative of a polar cluster. The increase of transverse correlation, which is a direct indicator of domain growth, surpasses the time scale of the abrupt change of dynamics in the system, measured by means of time autocorrelation. The critical slowing down and regime change takes place between 8 and 13 ps ( 5 ps). At the crossover, the system transforms from floppy-dynamic (polar clusters) into 

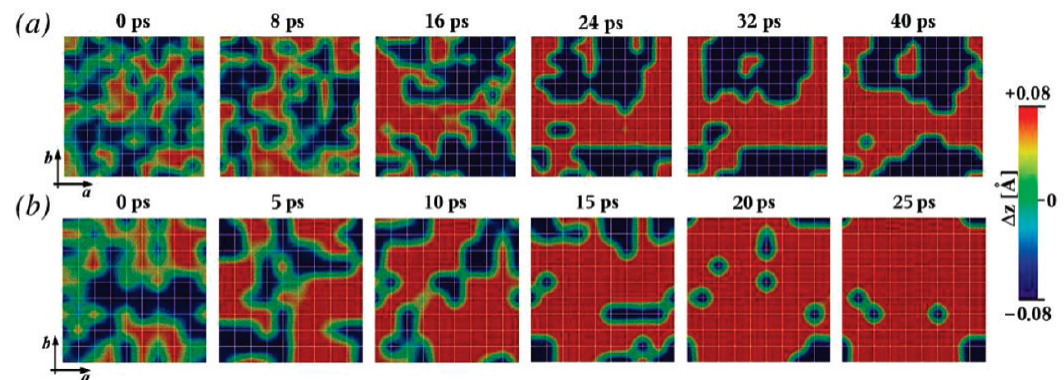

Figure 1. Transformation path for the PE to FE phase transition as induced by (a) temperature $\left(T=T_{\mathrm{c}}, 12^{3}\right.$ unit cells box) and (b) external field ( $9^{3}$ unit cells box). Ti displacements $\Delta z$ are shown in red and blue for up and down domain polarization, respectively. (a) Clustering is clearly visible in the cubic phase $(0-8 \mathrm{ps})$, just before domain growth onset $(8-16 \mathrm{ps})$. (b) Under a static electric field $\varepsilon(60 \mathrm{kV} / \mathrm{cm})$, the transformation rate is slower, such that certain chains remain antiparallel for a relatively long time ( $\geq 25 \mathrm{ps}$ ).

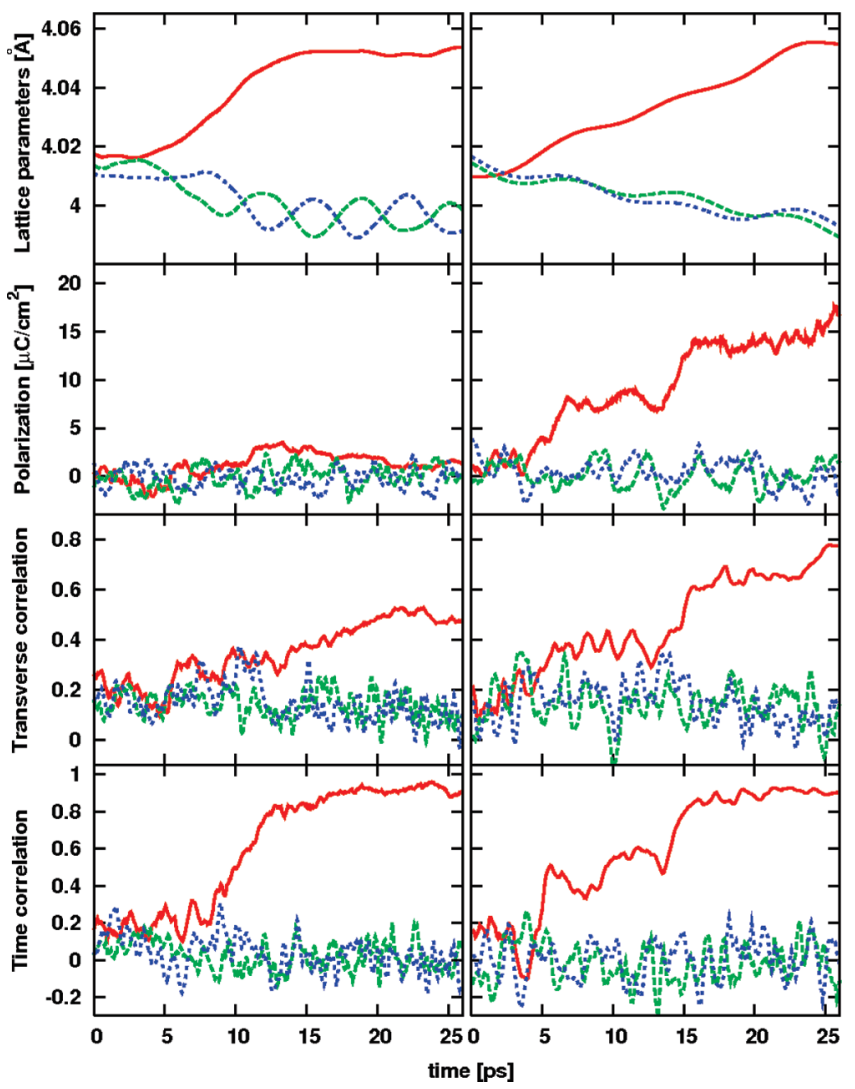

Figure 2. Change of the structural properties monitored by different order parameters. Left and right columns correspond to temperature (Figure 1a)- and field (Figure 1b)-induced PE to FE phase transition, respectively. Colors: blue, green, and red correspond to the $x, y$, and $z$ component, respectively. Time correlation is the chain autocorrelation $(0.5 \mathrm{ps})$. Note that domain growth (transverse correlation) surpasses the time scale of chain mobility freezing.

almost static (domains) under rescaling of critical lengths (in the $x, y$ nonpolar directions the system remains dynamic). While the PE phase is floppy and may host distant, time-decorrelated polar clusters (coarse and mobile domain boundaries), FE domain formation is a slower process, with long-ranged correlation lengths within domains and a changed chain-flip response (narrow and rigid domain walls). Since the rescaling does not affect the whole material at the same time, on the contrary it is taking place locally and asynchronously, narrow AFE islands (narrow islands of antiparallel chains) may remain trapped within larger domains (Figure 1a, 24, 32, 40 ps). This characteristic effect reflects an intrinsic property of BTO to accumulate 'AFE defects' within FE domains.
3.2. Chains and Ferrodomain Growth under an External Static Field. It is well-known that the ferroelectric transition temperature is affected by an external electric field. Along this line we have performed another set of TPSMD simulations under an external static electric field $\varepsilon$ along $z$, in order to collect further details on the dynamics of polar clusters and on the overall transition timing. Since the abrupt introduction of a static field on trajectories containing the transition may spoil the dynamics, different field values were initially applied to the two transition branches (very large value for $c \rightarrow t$, tiny for $t \rightarrow c$ ). Finite field value reduction $(c \rightarrow t)$ or enhancements $(t \rightarrow c)$ were iteratively incorporated into TPSMD, until an equal value of $60 \mathrm{kV} / \mathrm{cm}$ was reached for both branches. The corresponding changes in the order parameters are presented in Figure 2, right column. The $P_{z}$ component of the polarization increases up to a value of about $17.5 \mu \mathrm{C} / \mathrm{cm}^{2}$, close to the experimental value of $17.0 \mu \mathrm{C} / \mathrm{cm}^{2}{ }^{39}$ Both transverse correlation and time autocorrelation of chain growth indicate an overall slowing down of the cluster dynamics, like that observed for the field-free case, in the range 5-15 ps. However, the cluster freezing is slower by a factor two (10 ps vs 5 ps without field) under the effect of a field than in its absence. This effect ${ }^{25}$ is due to dynamical molecular field of enlarged clusters (Figure 1b, 0-10 ps), which effectively reduces cluster temperature and persistency.

3.3. Transition State, Phase Coexistence, and Symmetry Breaking Step. On the atomistic scale of our simulations, the mutual influence of pretransitional clusters due to size and polarization manifests itself in even subtler effects. The discontinuous nature of the transition leads to coexistence of (metastable) paraelectric and (already transformed) ferroelectric regions. Mixed configurations of paraelectric/ferroelectric BTO may show a momentary imbalance between oppositely polarized regions. To better illustrate this point, we present in Figure 3 snapshots of intermediate configurations between 4.5 and 9.8 ps (same time scale as in Figure 2) selected from the field-free transition. In this region, transverse correlation indicates size rescaling, which is enhanced under the effect of a field. Using a volumetric representation, we display regions of positive polarization in orange, separated from regions with opposite polarization (transparent) by a boundary (black). The shape of polar clusters in the paraelectric phase is unsteady (Figure 3a,b), as the boundary ceaselessly varies. A characteristic cluster shape in this region can hardly be defined. The amount of orange regions fairly fill half of the box, for a resulting vanishing polarization.

Starting at $6.7 \mathrm{ps}$, a portion at the center of the simulation box shows a less volatile shape, in the form of a durable polarized cluster, while the (black) boundary becomes sharper and persistent (7.2, 7.7 ps, Figure 3c,d). Next to this area, floppy 


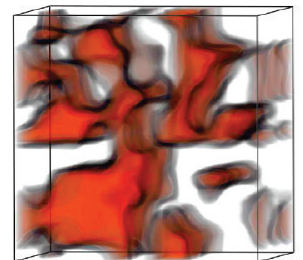

(a) $\quad 4.5 \mathrm{ps}$

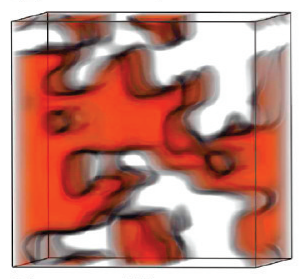

(e)

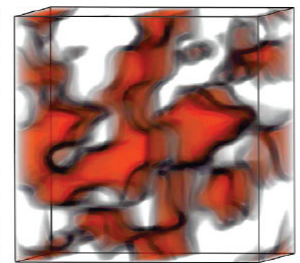

(b) $\quad 5.6 \mathrm{ps}$

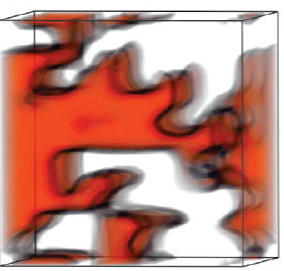

(f)

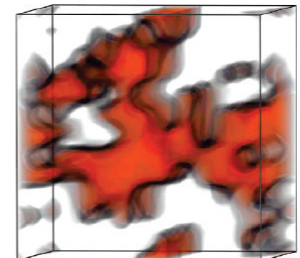

(c) $\quad 6.7 \mathrm{ps}$

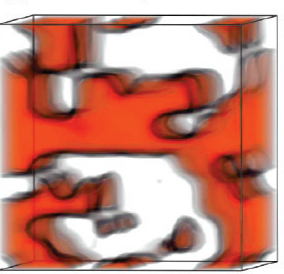

(g)

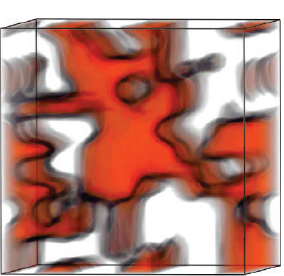

(d) $\quad 7.2 \mathrm{ps}$

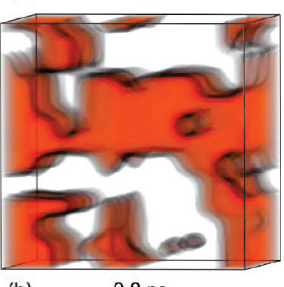

(h) $\quad 9.8 \mathrm{ps}$

Figure 3. Evolution from the paraelectric state $(\mathrm{a}, \mathrm{b})$, characterized by polarized, albeit floppy, clusters (4.5-6.7 ps), into a ferroelectric regime (e). Intermediate configurations (c, d) feature the coexistence of paraelectric and ferroelectric regions. A sharp and durable domain boundary between paraelectric and ferroelectric regions is formed upon slowing of the chain flipping (7.2 ps).

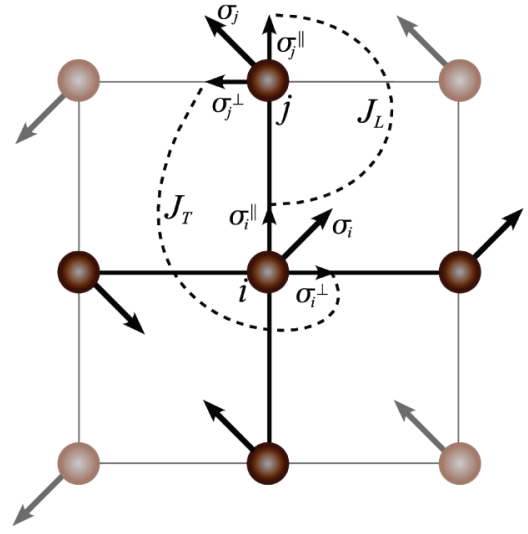

Figure 4. Two-dimensional interaction diagram for the Hamiltonian in eq 1. The labeling is explained in the text. Nearest neighbors of atom $i$ are in full color.

polar clusters of the paraelectric state are still present. This hardening of the boundary distinguishes this intermediate stage of coexisting paraelectric and ferroelectric phenomena, which is also reflected by the large variation of the time autocorrelation (Figure 2).

The persistence of a boundary on one hand and a volatile cluster behavior on the other indicate disparate chain-flipping dynamics and unequal coherence lengths of dipole alignments. As a consequence, an imbalance in the overall polarization can be realized, which follows from local decoupling time and length scales in different fashions, as a funtion of the space coordinate.

Since the paraelectric and ferroelectric phenomena are topographically distinguishable by a hardened boundary, which is clearly marking the departure from a "pure" paraelectric situation of fluctuating polar custers (the pretransitional step), we identify this step as the symmetry breaking event. A representative transition state for the paraelectric to ferroelectric phase transition of BTO is the one depicted in Figure 3, 7.2 ps.

The transverse correlation reveals further domain growth after 20 ps. A purely electrostrictive scenario would imply growth of one domain at the expense of the other after $10 \mathrm{ps}$ under the effect of the field. In BTO, however, the tendency of placing antiparallel chains (AFE defects) within large FE domains is retained and survives in the mature, single domain BTO after $25 \mathrm{ps}$, to the extent that the final steps are only affected by AF chain dynamics. Due to the marked slowing of the dynamics of chain flipping within domains, AF defects, albeit metastable, (a)
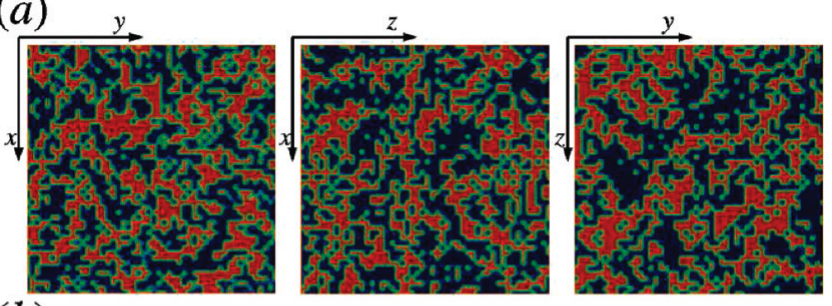

(b)
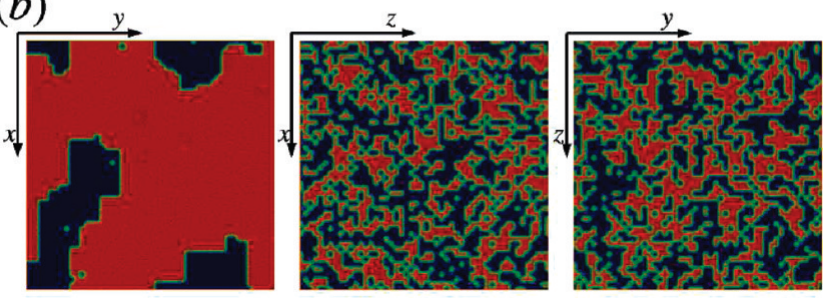

(c)
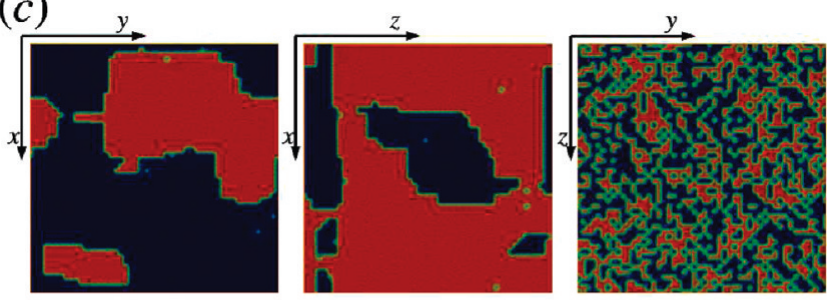

(d)
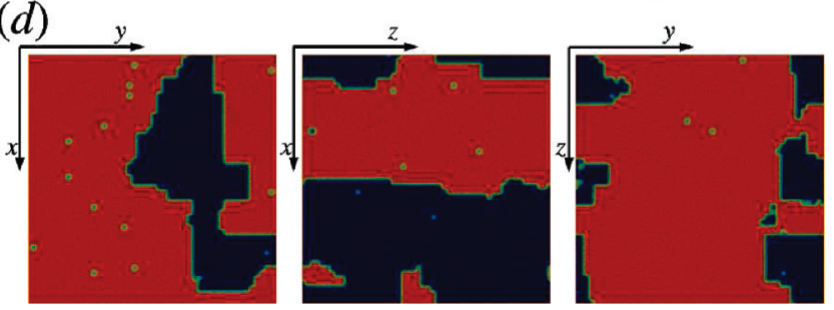

Figure 5. Results of MC simulation for the spin-like model. Twodimensional mutually perpendicular sections are presented. Colors denote the direction of the Ti shift $(\boldsymbol{\sigma})$ component perpendicular to the plane of the section: red, up; blue, down. The parameters are as follows: $(\mathrm{a}-\mathrm{c}) J_{\mathrm{T}}=8.45 \times 10^{-2} J_{\mathrm{L}}$ and $k T=0.644 J_{\mathrm{L}}$, (d) $J_{\mathrm{T}}=8.45 \times 10^{-2} J_{\mathrm{L}}$ and $k T=0.4 J_{\mathrm{L}}$. The directions of $\boldsymbol{\sigma}$ : (a) $\boldsymbol{\sigma} \in\{\langle 111\rangle\}$, (b) $\boldsymbol{\sigma}$ deviating from $\langle 111\rangle$ by $12.5^{\circ}$ toward [00 \pm 1$]$, (c) $\sigma$ deviating from $\langle 111\rangle$ by $4.5^{\circ}$ toward $[0 \pm 1 \pm 1],(\mathrm{d}) \boldsymbol{\sigma} \in\{\langle 111\rangle\}$.

can survive over a long period of time (on the scale of the simulation) and are expected to play a role in the switching response of the material. 

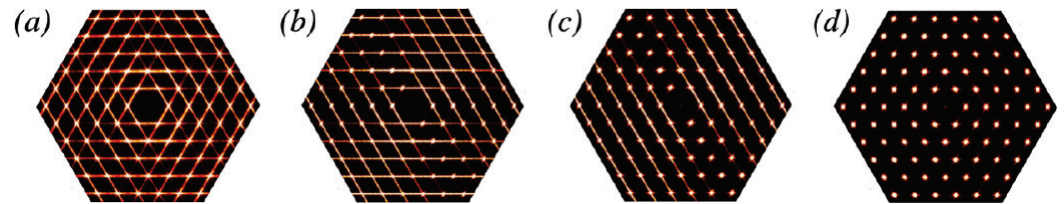

Figure 6. Diffuse scattering on the (111) planes in reciprocal space for different phases of BTO: (a) cubic, (b) tetragonal, (c) orthorhombic, and (d) rhombohedral. The successive ordering is evidenced by the disappearance of diffuse lines.
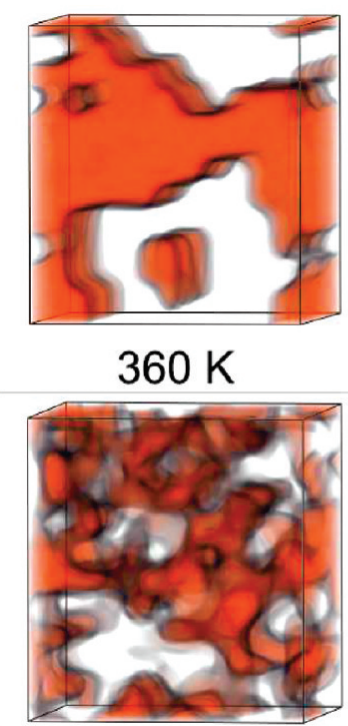

$480 \mathrm{~K}$

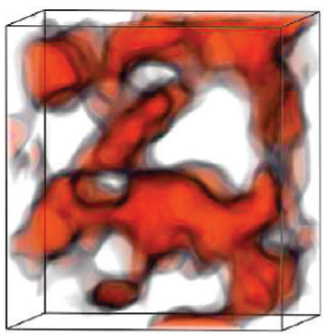

$380 \mathrm{~K}$

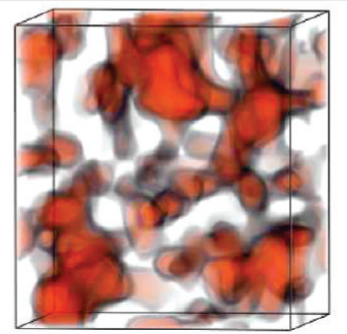

$520 \mathrm{~K}$

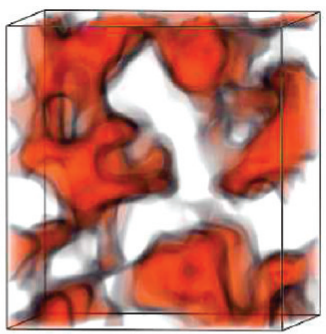

$440 \mathrm{~K}$

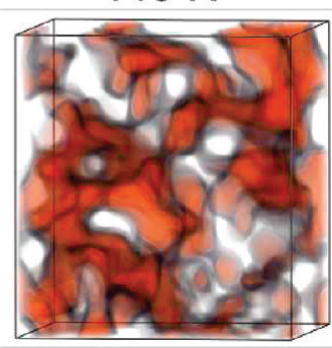

$560 \mathrm{~K}$

Figure 7. 3D volumetric representation of clustering at different temperatures. Regions of positive polarization are represented in orange (negative polarization is transparent). The boundaries are black. Above $440 \mathrm{~K}$, the size and time stability of the clusters markedly change. For comparison, a configuration obtained at $360 \mathrm{~K}$ showing ferroelectric domains is presented on the left.

3.4. Mesoscale Domain Structure. The picosecond-resolved chain-flip and clustering events accessible through MD dynamics represent key dynamical aspects of the PE to FE phase transition in BTO. To coarse-grain the atomistic findings, the highly structured vector field of $\mathrm{Ti}-\mathrm{O}$ dipoles (Figure 1) was mapped onto a Heisenberg spin-like model, allowing for easily upscaling the system, in order to capture mesoscale domains. Along the experience of a previous work, ${ }^{40}$ we designed the spin Hamiltonian with explicit reference to correleted chain displacements.

The local mode $\boldsymbol{\sigma}$ is defined as displacement of $\mathrm{Ti}$ atoms from the center of the oxygen octahedra, which are structured into chains $(\mathrm{Ti}-\mathrm{O})_{n}$ with a large longitudinal correlation. Transverse correlation leads to the formation of nanoclusters. A model of correlation of $\boldsymbol{\sigma}$ as a bilinear form can be postulated:

$$
H=-\sum_{\langle i, j\rangle}\left[J_{L} \sigma_{i}^{\prime \prime} \sigma_{j}^{ل !}+J_{T}\left(\sigma_{i}^{\perp 1} \sigma_{j}^{\perp 1}+\sigma_{i}^{\perp 2} \sigma_{j}^{\perp 2}\right)\right]
$$

where $\langle i, j\rangle$ stands for the pair of nearest neighbors unit cells, $\sigma^{\Perp}, \sigma^{\perp 1}$, and $\sigma^{\perp 2}$ are the components of the displacement vector $\boldsymbol{\sigma}$ parallel and perpendicular to the direction of the $i-j$ bond, respectively. $\boldsymbol{\sigma} \in\{\langle 111\rangle\}$ accounts for the minima in the diagonal pseudocubic directions. ${ }^{18,41,42}$ For $J_{\mathrm{T}}=0$, the model breaks into noncoupled Ising chains of the eight-site model. ${ }^{43}$ The parameters $J_{\mathrm{L}}$ and $J_{\mathrm{T}}$ were fitted to MD simulations of the cubic paraelectric phase only, yielding the relations: $J_{\mathrm{T}}=8.45$ $\times 10^{-2} J_{\mathrm{L}}$ for $k T=0.644 J_{\mathrm{L}}$. The resulting domain structures are shown in Figure 5a. The disordered, yet on the average, cubic structure features polar nanoclusters and represents the upscaled version of the features of Figure 1.
However, can model parameters fitted on the cubic phase account for all other phases? In tetragonal BTO, $\boldsymbol{\sigma}$ deviates from $\langle 111\rangle$ by $12.5^{\circ}(\mathrm{MD})$ or $11.8^{\circ}\left(\mathrm{EXAFS}^{42}\right)$ toward $\langle 001\rangle$. Performing $\mathrm{MC}$ simulations with $\boldsymbol{\sigma} \quad\left(\sigma_{z} \rightarrow 1.063 \sigma_{z}\right.$ and $\left.\sigma_{x, y} \rightarrow 0.967 \sigma_{x, y}\right)$ results in the configuration of Figure $5 \mathrm{~b}$. This parameter choice stabilizes the tetragonal phase. In the orthorhombic phase shift directions deviate from $\langle 111\rangle$ by $4.5^{\circ}$ toward $\langle 011\rangle$ (as obtained from low-temperature MD simulations not presented in this paper). Indeed, displacement vectors $\sigma_{z, y} \rightarrow 1.052 \sigma_{z, y}$ and $\sigma_{x} \rightarrow 0.885 \sigma_{x}$ generate the orthorhombic phase (Figure 5c). For the transformation to the rhombohedral phase (Figure 5d), the initial set of directions $\langle 111\rangle$ can be used with a changed MC simulation temperature. The spin-like model for $\boldsymbol{\sigma} \in\{\langle 111\rangle\}$ fully describes the cubic-to-rhombohedral phase transition. In general, the same interaction parameters fitted on the cubic phase yield different phases depending on the form of $\boldsymbol{\sigma}$. This validates the parametrization step.

3.5. Diffuse Scattering. The measurement and interpretation of diffuse scattering remains an involved task. ${ }^{44}$ In the case of BTO, calculating DS is particularly important for assessing the reliable description of the complicated correlations of $\mathrm{Ti}$ displacements along the transitions, which is captured by the MD layer and transferred onto the MC one, like described above. In Figure 6, diffuse scattering patterns calculated for different phases are presented. Cubic, tetragonal, and orthorhombic structures show DS features along 3,2, and only 1 (111) plane, reflecting the ordering features in orthogonal planes displayed in Figure 5. The DS planes result from strong longitudinal correlation of displacements within $(\mathrm{Ti}-\mathrm{O})_{n}$ chains, which set in along a different direction as a function of temperature. The transition from one phase to the other is clearly evidenced by 
the extinction of one set of diffuse lines (planes). The scalingup procedure, from MD to MC, is essential for good DS pattern resolution. Accordingly, our results are in excellent agreement with experiments ${ }^{43}$ and with recent calculations ${ }^{38}$ based on DFT. Apart from further validation of the MD/MC layers, this clearly indicates the common nature of the dynamical chain ordering and size/time rescaling over all phase transitions.

3.6. Clusters Dynamics. Burns and $D^{2}{ }^{21}$ suggested that polarized clusters exist even at $200 \mathrm{~K}$ above the Curie temperature, as determined by refractive index measurements on cubic $\mathrm{BaTiO}_{3}$. In the attempt to visualize clustering as a function of temperature, and to estimate a temperature of transition into the clustering regime, we have performed simulations on the cubic phase in the temperature range 380-560 K. Calculations were started from the critical trajectories of Figure 1. Applying shift and shoot moves during TPS simulations ensures a progressive optimization of polarized clusters at the desired temperature without any constraint or bias on either internal or mutual cluster interactions.

A representative cluster landscape for different temperatures is displayed in Figure 7. As for the transition at the Curie temperature, we used transverse correlation to define a transition temperature into the clustering regime $\left(T_{\text {clus }}\right)$. Above $T_{\text {clus }}=440$ $\mathrm{K}$, its value drops below 0.1 , indicating a significant loss of structuring in the $x, y$ plane. Apart from an overall fragmentation and size reduction, clusters survive above $T_{\text {clus }}$ for less than 100 fs. Despite large size fluctuations, no further cluster and polarization growth is observed in this regime. $T_{\text {clus }}$ is in the range of experimental determinations..$^{21,22,45}$

\section{Conclusions}

In conclusion, we have presented an atomistic investigation of the paraelectric to ferroelectric phase transition in BTO. Dynamical polar pretransitional clusters, already present in the cubic paraelectric phase, grow into larger ferroelectric nanodomains while the $\mathrm{Ti}-\mathrm{O}$ dipole dynamics slow down. The transition state is characterized by coexisting paraelectric and ferroelectric phenomena, separated by a persistent phase boundary, different from the soft, frail boundary of pretransitional polar clusters in the paraelectric phase. Like in relaxors, local polar moments hint at localized phonons. ${ }^{46,47}$ Unlike relaxors, size rescaling in BTO restores lattice dynamics more close to perfect ordered crystals (with the rhombohedral phase as the limiting case of perfect dipole order), while in relaxors only the slowing process assists the formation of mesoscopic regions with local polarization. Along this line, the local size and time rescaling resulting from our TPSMD simulations, together with the detailed cluster dynamics and growth mechanism, represents a general scheme for thinking about ordering phenomena in classic and relaxor ferroelectrics.

Acknowledgment. M.P. acknowledges the Polish Ministry of Education and Science (grant no. N N202 023334), Australian Research Council, and the IMPRS, Dresden. We thank ZIH, TU-Dresden, for computational time.

\section{References and Notes}

(1) Spaldin, N. A. Science 2004, 304, 1606.

(2) Nunez, M.; Buongiorno Nardelli, M. Phys. Rev. Lett. 2008, 101 107603

(3) Ma, S.; Wang, X.; Zhou, J.; Li, L.; Sun, C. Q. J. Appl. Phys. 2010, 107, 064102

(4) Fong, D. D.; Stephenson, G. B.; Streiffer, S. K.; Eastman, J. A.; Auciello, O.; Fuoss, P. H.; Thompson, C. Science 2004, 304, 1650.
(5) Cochran, W. Phys. Rev. Lett. 1959, 3, 412.

(6) Comes, R.; Lambert, M.; Guinier, A. Solid State Commun. 1968, 6,715 .

(7) Bersuker, I. B. Phys. Lett. 1966, 20, 589.

(8) Chaves, A. S.; Barreto, F. C. S.; Nogueira, R. A. Phys. Rev. B 1976, 13, 207.

(9) Lines, M. E.; Glass, A. M. Principles and Applications of Ferroelectrics and Related Materials; Clarendon Press: Oxford, 1977; pp 398-407.

(10) Harada, J.; Axe, J. D.; Shirane, G. Phys. Rev. B 1971, 4, 155.

(11) Bussmann-Holder, A.; Beige, H.; Völkel, G. Phys. Rev. B 2009, $79,184111$.

(12) Stern, E. A. Phys. Rev. Lett. 2004, 93, 037601.

(13) Zalar, B.; Laguta, V. V.; Blinc, R. Phys. Rev. Lett. 2003, 90, 037601.

(14) Ponomareva, I.; Bellaiche, L.; Ostapchuk, T.; Hlinka, J.; Petzelt,

J. Phys. Rev. B 2008, 77, 012102 .

(15) Hlinka, J.; Ostapchuk, T.; Nuzhnyy, D.; Petzelt, J.; Kuzel, P.; Kadlec, C.; Vanek, P.; Ponomareva, I.; Bellaiche, L. Phys. Rev. Lett. 2008, 101,167402 .

(16) Jesse, S.; Rodriguez, B. J.; Choudhury, S.; Baddorf, A. P.; Vrejoiu, I.; Hesse, D.; Alexe, M.; Eliseev, E. A.; Morozovska, A. N.; Zhang, J.; Chen, L. Q.; Kalinin, S. V. Nat. Mater. 2008, 7, 209.

(17) Maksymovych, P.; Jesse, S.; Huijben, M.; Ramesh, R.; Morozovska, A.; Choudhury, S.; Chen, L. Q.; Baddorf, A. P.; Kalinin, S. V. Phys. Rev. Lett. 2009, 102, 017601 .

(18) Tinte, S.; Stachiotti, M. G.; Sepliarsky, M.; Migoni, R. L.; Rodriguez, C. O. Ferroelectrics 2000, 237, 345.

(19) Krakauer, H.; Yu, R. C.; Wang, C. Z.; Rabe, K. M.; Waghmare, U. V. J. Phys. Condens. Matter 1999, 11, 3779.

(20) Yu, R.; Krakauer, H. Phys. Rev. Lett. 1995, 74, 4067.

(21) Burns, G.; Dacol, F. H. Solid State Commun. 1982, $42,9$.

(22) Dul'kin, E.; Petzelt, J.; Kamba, S.; Mojaev, E.; Roth, M. Appl. Phys. Lett. 2010, 97, 032903.

(23) Ziebińska, A.; Rytz, D.; Szot, K.; Górny, M.; Roleder, K. J. Phys. Condens. Matter 2008, 20, 142202.

(24) Tai, R. Z.; Namikawa, K.; Sawada, A.; Kishimoto, M.; Tanaka, M.; Lu, P.; Nagashima, K.; Maruyama, H.; Ando, M. Phys. Rev. Lett. 2004, 93, 087601.

(25) Namikawa, K.; Kishimoto, M.; Nasu, K.; Matsushita, E.; Tai, R. Z.; Sukegawa, K.; Yamatani, H.; Hasegawa, H.; Nishikino, M.; Tanaka, M.; Nagashima, K. Phys. Rev. Lett. 2009, 103, 197401.

(26) Sepliarsky, M.; Migoni, R. L.; Stachiotti, M. G. Comput. Mater. Sci. 1998, 10, 51 .

(27) Edwardson, P. J. Phys. Rev. Lett. 1989, 63, 55.

(28) Bolhuis, P. G.; Dellago, C.; Chandler, D. Faraday Discuss. 1998, $110,421$.

(29) Dellago, C.; Bolhuis, P. G.; Geissler, P. Lect. Notes Phys. 2006, 703,349 .

(30) Zahn, D.; Leoni, S. Phys. Rev. Lett. 2004, 92, 250201

(31) Boulfelfel, S. E.; Zahn, D.; Grin, Y.; Leoni, S. Phys. Rev. Lett. 2007, 99, 125505 .

(32) Leoni, S.; Ramlau, R.; Meier, K.; Schmidt, M.; Schwarz, U. Proc. Natl. Acad. Sci. U.S.A. 2008, 105, 19612.

(33) Smith, W.; Forester, T. J. J. Mol. Graphics 1996, 14, 136.

(34) Sepliarsky, M.; Asthagiri, A.; Phillpot, S. R.; Stachiotti, M. G.;

Migoni, R. L. Curr. Opin. Solid State Mater. Sci. 2005, 9, 107.

(35) Metropolis, N.; Rosenbluth, A. W.; Rosenbluth, M. N.; Teller,

A. H.; Teller, E. J. Chem. Phys. 1953, 21, 1087.

(36) Wolff, U. Phys. Rev. Lett. 1989, 62, 361.

(37) Proffen, T.; Neder, R. B. J. Appl. Crystallogr. 1997, 30, 171.

(38) Zhang, Q.; Cagin, T.; Goddard, W. A., III. Proc. Natl. Acad. Sci. U.S.A. 2006, 103, 14695.

(39) Kwei, G. H.; Lawson, A. C.; Billinge, S. J. L.; Cheong, S. W. J. Phys. Chem. 1993, 97, 2368.

(40) Paściak, M.; Wołcyrz, M.; Pietraszko, A.; Leoni, S. Phys. Rev. B 2010, 81, 014107 .

(41) Cohen, R. E. Nature 1992, 358, 136.

(42) Ravel, B.; Stern, E. A.; Vedrinskii, R. I.; Kraizman, V. Ferroelectrics 1998, 206, 407.

(43) Comes, R.; Lambert, M.; Guinier, A. Acta Crystallogr., Sect. A: Found. Crystallogr. 1970, 26, 244.

(44) Ravy, S.; Itié, J. P.; Polian, A.; Hanfland, M. Phys. Rev. Lett. 2007, 99, 117601.

(45) Rusek, K.; Kruczek, J.; Szot, K. Ferroelectrics 2008, 375, 165.

(46) Dmowski, W.; Vakhrushev, S. B.; Jeong, I. K.; Hehlen, M. P.; Trouw, F.; Egami, T. Phys. Rev. Lett. 2008, 100, 137602.

(47) Liu, Y.; Withers, R. L.; Nguyen, B.; Elliott, K. Appl. Phys. Lett. 2007, 91, 152907.

JP106206Y 